\title{
Randomised trials of STD treatment for HIV prevention: report of an international workshop
}

\section{Workshop}

Richard Hayes, Maria Wawer, Ron Gray, James Whitworth, Heiner Grosskurth, David Mabey, and HIV/STD Trials Workshop Group*

Three community trials of the impact of STD treatment interventions on HIV incidence in rural populations have been completed or are in progress in Uganda and Tanzania. Investigators from these trials met for a joint technical workshop in Baltimore in May 1996. This report summarises the consensus of the workshop, with the aim of providing useful input to research on HIV intervention strategies. Issues discussed include: (i) the role of community randomised trials; (ii) strategies for STD management; (iii) epidemiological and statistical issues in the design and analysis of community randomised trials; (iv) diagnostic methods for STDs in population surveys; (v) treatment regimens for STDs in rural Africa; and (vi) ethical issues in community trials.

(Genitourin Med 1997;73:432-443)

Keywords: community trials; STD treatment: HIV prevention

\section{Introduction}

The HIV epidemic continues to expand rapidly in many parts of the world and more than three million new infections are estimated to have occurred during 1995 , over $95 \%$ of them in developing countries. Sub-Saharan Africa continues to be the most severely affected region, but rapid increases are also being recorded in many parts of southern Asia. ${ }^{1}$ Heterosexual transmission predominates in these regions, and has been estimated to account for more than $90 \%$ of adult infections in Africa. ${ }^{2}$ To date, population based control programmes have focused primarily on behavioural interventions intended to reduce sexual transmission but achieving rapid, substantial, and sustained changes in sexual behaviour is difficult. The World Health Organisation (WHO) has advocated complementing behavioural interventions with improved treatment services for the classic sexually transmitted diseases (STDs) such as gonorrhoea, syphilis, chancroid, and chlamydia. The sexual transmission of HIV is thought to be enhanced in the presence of such STDs, ${ }^{34}$ which are highly prevalent in many parts of the developing world due at least partly to inadequate treatment services. ${ }^{5}$ It has been suggested that the treatment of bacterial STDs may be one of the most cost effective health interventions available in some developing countries. ${ }^{67}$

Well designed randomised trials are recognised as the gold standard for the evaluation of public health interventions, and it is important that such trials are conducted to provide reliable data on the efficacy and cost effectiveness of control strategies, in order to provide a sound basis for health policy and resource allocation. No randomised trials of the impact of behavioural interventions on HIV incidence have yet been reported. The first randomised trial of improved STD treatment was com- pleted in Mwanza Region, Tanzania in 1995 demonstrating a $42 \%$ reduction in HIV incidence among adults in the general rural population, ${ }^{8}$ and two further randomised trials are now in progress in east Africa. A trial in Rakai District, Uganda, is investigating the impact of intensive STD control via periodic mass treatment for STDs among adults in the general population. Nearby, in Masaka District, Uganda, a further trial is comparing groups receiving information, education, and communication (IEC); IEC together with improved syndromic treatment services for STDs; and a comparison group receiving a community development intervention.

Investigators from these three trials met at Johns Hopkins University, Baltimore, United States in May 1996 in a joint technical workshop. The purpose of this report is to summarise the consensus of the workshop, with the aim of providing useful input to research on HIV intervention strategies. The report summarises key issues and conclusions concerning (i) the role of community randomised trials; (ii) strategies for STD management; (iii) epidemiological and statistical issues in the design and analysis of community randomised trials; (iv) diagnostic methods for STDs in population surveys; (v) treatment regimens for STDs in rural Africa; and (vi) ethical issues in community trials.

The role of community randomised trials STD control for HIV prevention is an unusual epidemiological strategy in which control of one set of diseases is used to reduce the incidence of another. In all three African trials, the impact of this strategy is being measured directly by randomising entire communities either to an STD intervention arm or to a comparison arm. The main reasons for adopting community randomisation are as follows.

\author{
end of the article. \\ Correspondence to: \\ Disease Epidemiology Unit, \\ London School of Hygiene $\&$ \\ Street, London WC1E 7HT. \\ Accepted for publication \\ 28 August 1997
}


CONTROL OF CONFOUNDING

There is extensive evidence from observational studies that STDs are associated with HIV infection, ${ }^{34}$ but concerns have been raised that these associations may not be causal, and may reflect confounding due to sexual behaviours that are risk factors both for STDs and for HIV. Randomised trials provide stronger evidence of a causal association, since random allocation of the intervention (STD control) helps to avoid confounding by enhancing the comparability of intervention and comparison groups. ${ }^{9}$

COMMUNITY RANDOMISED TRIALS MEASURE MAXIMAL IMPACT ON HIV INCIDENCE

In the Mwanza and Masaka trials, the choice of intervention strategy (improved treatment services at community health units) dictated the randomisation of entire communities. In the Rakai project, all adults in the intervention arm are offered single dose oral treatment for STDs, whether or not they are symptomatic.

STDs are thought to enhance HIV incidence by increasing both the infectiousness of HIV infected individuals and the susceptibility of HIV uninfected individuals. The impact of STD control could, in principle, be tested by individually randomising HIV negative subjects to an STD intervention or to a comparison arm. However, this design would fail to capture the full impact of the intervention when applied at population level, since it would measure only the effect of STD control on HIV acquisition, and since any such effects may be overwhelmed by rapid STD reinfection of treated trial subjects by their untreated partners. STD control in the entire population, as in a community randomised trial, may have a greater impact on HIV incidence since the intervention operates both on HIV acquisition ("direct effect") and transmission ("indirect effect"), and this design captures the "mass effect" on transmission achieved when most members of a community are covered by an intervention.

\section{COMMUNITY RANDOMISED TRIALS MINIMISE CONTAMINATION}

The term "contamination" in this context refers principally to the incidence of HIV or STD infections among intervention arm subjects through sexual contact with infected individuals from outside the treated population. Rapid STD reinfection is a serious concern in individually randomised trials, but can be minimised in community randomised trials by defining communities to coincide with predominant sexual networks. In the Rakai trial, for example, preliminary studies were carried out to map geographical patterns of social interaction.

Where improved treatment services are provided through health units, contamination may also occur if comparison arm subjects gain access to the intervention health units. This effect can again be minimised by careful definition of communities, and can be monitored by recording the residence of clinic attenders. In the Mwanza and Masaka trials, the geographical separation of intervention and comparison communities precluded ready access of comparison subjects to the improved services.

Key messages
- Randomised intervention trials provide the
strongest evidence of STD cofactor effects.
- Community randomisation captures
effects of STDs on both HIV acquisition
and infectiousness.
- Community randomisation captures the
"mass effect" of an intervention.
- Community randomisation minimises
contamination of intervention and control
groups, providing the communities are
carefully selected.

\section{Strategies for STD treatment}

The main aim of the three African trials is to measure the impact of improved treatment services for STDs on the incidence of HIV infection in the general adult population. In this section, we discuss the various strategies for STD treatment that might be investigated.

\section{THE PIOT-FRANSEN PYRAMID}

The discussion is illustrated by reference to the Piot-Fransen model of STD management (Fig 1A), which shows the steps necessary for each STD to be effectively treated, and the estimated success rate achieved at each step. The pyramid shown is typical of rural women in many parts of Africa, and is based on data from Uganda, Zaire, and Tanzania.

About $50 \%$ of all sexually active women in the community have a reproductive tract infection (RTI) at any point in time, but only about half of these are symptomatic. ${ }^{10}$ Little more than half of the symptomatic women seek treatment, and not all of these go to a health unit or other medically trained care provider. ${ }^{11} 12$ Of the $30 \%$ who may present for treatment, as few as one in five may be treated appropriately unless improved syndromic services are provided. Not all of these will complete their treatment, and not all will be cured. Of those cured, at most a third will persuade their partners to seek treatment.

Under these conditions, only $3 \%$ of infected women will be cured, and many of these may be rapidly reinfected because their partners remain untreated. It can be shown that the situation in males is little better, especially in view of recent data showing a high rate of asymptomatic infections in men. ${ }^{1314}$ These results help to explain the very high prevalence and incidence of STDs in many parts of Africa.

The pyramid can be used to consider the likely effects of alternative STD treatment strategies.

\section{SYNDROMIC TREATMENT SERVICES}

The Mwanza and Masaka trials investigate improved treatment services provided through existing primary health care units, based on syndromic treatment of STDs as recommended by the WHO. Data from Mwanza 
A

All women

With STD/RTI

Symptomatic

Seek treatment

Go to health unit

Treated correctly

Compliant

Treatment effective

Partner treated

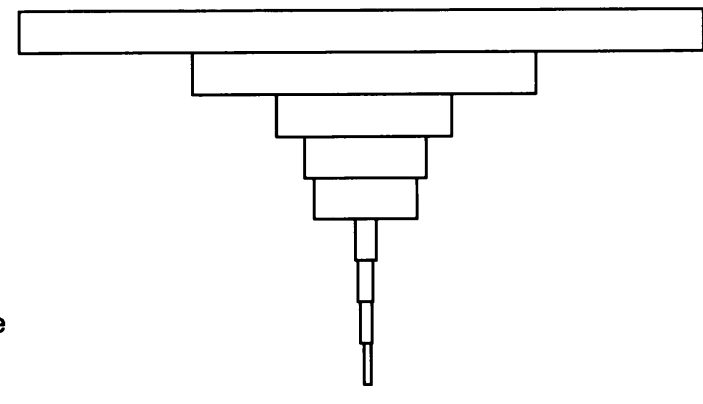

$100 \%$

$50 \%$

$35 \%$

$30 \%$

$6 \%$

$4 \%$

$3 \%$

$1 \%$

B

All women

With STD/RTI

Symptomatic

Seek treatment

Go to health unit

Treated correctly

Compliant

Treatment effective

Partner treated

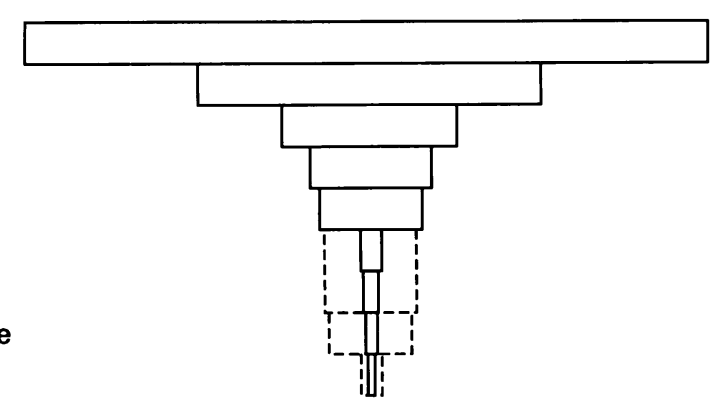

C

All women

With STD/RTI

Symptomatic

Seek treatment

Go to health unit

Treated correctly

Compliant

Treatment effective

Partner treated

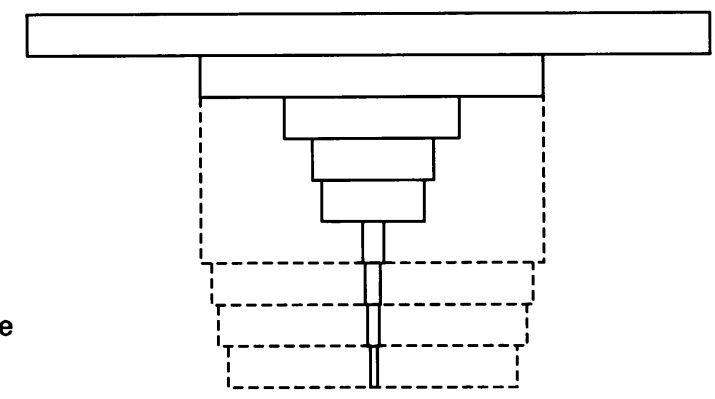

(A) Piot-Fransen model of STD management in rural women. The figure shows

$S T D / R T I$ prevalence in the community, the steps needed to ensure effective treatment, and the percentage of STD/RTI effectively treated. Data are illustrative and based on studies in Uganda, Zaire, and Tanzania. (B) Piot-Fransen model, showing potential effect of syndromic STD treatment services. (C) Piot-Fransen model, showing potential effect of mass STD treatment. may be particularly important in enhancing HIV transmission. However, it is clear that STD transmission will continue to be fuelled by asymptomatic or mildly symptomatic infections, and that more intensive treatment strategies may need to be considered if the substantial public health burden associated with STDs is to be addressed, and if the impact against $\mathrm{HIV}$ is to be maximised and sustained. Some additional strategies are considered below.

\section{SCREENING FOR SYMPTOMATIC BUT}

NEGLECTED CASES

The impact of treatment services could be increased by screening measures to detect symptomatic but neglected STD cases. Target groups might include individuals attending non-STD health services, such as antenatal, mother and child health, or family planning clinics. A short interview might be supplemented with a brief genital examination. However, both health workers and patients may find such measures invasive in a nonSTD setting. Furthermore, experience suggests that the proportion of symptomatic patients failing to come for treatment may decrease once improved services are in place.

\section{SCREENING FOR ASYMPTOMATIC CASES}

A larger impact might be expected through measures to identify and treat asymptomatically infected individuals. Such strategies, however, would be expensive, logistically difficult, invasive, and subject to the limitations of available screening and diagnostic tests. Risk factors, such as young age and single marital status, have been used to develop risk scores to predict the likelihood of STD infection, with the aim of treating individuals with a high score. However, such risk scores need to be tailored to the local situation, and most have shown low sensitivity and predictive value. ${ }^{1017}$ The greatest burden of STDs falls on pregnant women and their offspring. Further research is needed on strategies for screening antenatal clinic attenders, both for syphilis and other STDs, and for treating their partners. However, the overall impact of such measures on HIV transmission is limited by the relatively long intervals between pregnancies, the fact that many women do not receive antenatal care or only receive care late in pregnancy, and the difficulty of reaching partners.

STD CONTROL IN HIGH RISK GROUPS

The control of STDs in high risk groups, such as commercial sex workers and truck drivers, may be a particularly cost effective strategy. ${ }^{618}$ Research on innovative approaches, such as "women's centres" situated in red light districts, is urgently needed. In rural areas, however, high risk groups are generally difficult to define and identify. For this reason, the three African trials have all adopted strategies aimed at the general population.

STD CONTROL THROUGH MASS TREATMENT

Mass treatment involves the treatment of the entire sexually active population, ideally over a attenders. The substantial reduction in HIV incidence suggests that symptomatic STDs 
large area and within a short period of time. The objective is to achieve an immediate reduction in the prevalence (and hence incidence) of STDs to a low level. It can be applied on one occasion or periodically, depending on the rate at which STDs are reintroduced. The Rakai trial is investigating the impact of repeated mass treatment at approximately 9 monthly intervals.

In principle, mass treatment addresses all the main limitations of other strategies (Fig 1C). Asymptomatic infections are treated, infected partners are covered without the need for contact tracing, treatment seeking behaviour is not required, and compliance and treatment effectiveness can be assured through the use of a directly observed single dose oral regimen of proved efficacy. Mass treatment is unlikely to be cheap, but may be highly cost effective if the impact on HIV and other STDs is substantial.

The approach is novel, controversial, and may not always be politically acceptable. There are also concerns about logistics, side effects, and the possible development of drug resistance. Nevertheless, the potential impact of this strategy on morbidity and mortality is so great that it should be evaluated in rigorously designed studies, including careful monitoring for adverse effects. Consideration should be given to evaluating a combined intervention, in which treatment services are improved and a single round of mass treatment is added to bring about an initial reduction in STD prevalence.

Key messages

- The Piot-Fransen pyramid can be used to analyse obstacles to effective STD treatment in any population.

- In many parts of rural Africa, existing health services treat effectively only a small proportion of all STDs.

- Improved syndromic treatment services increase the proportion of cases effectively treated, but their impact is limited by the high prevalence of asymptomatic STDs and the low proportion of partners treated.

- More intensive STD treatment strategies may be needed to further reduce STD related morbidity and HIV transmission.

- Screening for asymptomatic cases is limited by the lack of affordable and reliable diagnostic tests.

- STD control in high risk groups is cost effective, but may be ineffective in rural areas where such groups are difficult to identify and access.

- Mass treatment for STDs overcomes many of the problems associated with treatment of symptomatics, and should be rigorously evaluated.

\section{Epidemiological and statistical issues}

The design of community randomised trials of STD interventions requires careful consideration of sample size (number of units of randomisation and number of individuals within each unit), of methods to ensure comparability between study arms and to adjust for potential confounders, and of analyses to link any impact on HIV to effects on STDs.

\section{SAMPLE SIZE}

In all three of the east African trials, interventions were randomly allocated to entire communities rather than to individuals, since the aim was to measure the impact of intervening in a whole population. ${ }^{19}$ Standard sample size formulas for individually randomised trials ${ }^{20}$ have to be amended to take account of the additional random error resulting from between community variability.

An approximate estimate of $n$, the number of communities required in each arm of the trial, may be obtained as ${ }^{19}$ :

$$
\mathrm{n}=1+\frac{\mathrm{f}\left[\left(\lambda_{0}+\lambda_{1}\right) / \mathrm{y}+\mathrm{k}^{2}\left(\lambda_{0}^{2}+\lambda_{1}^{2}\right)\right]}{\left(\lambda_{0}-\lambda_{1}\right)^{2}}
$$

where $\lambda_{0}$ and $\lambda_{1}$ are the average incidence rates in the study communities in the absence and presence of the intervention, $y$ is the person years of observation in each community, $k$ is the coefficient of variation ( $\mathrm{SD} /$ mean) of the (true) incidence rates among the communities in each arm, and $f=10.51$ or 7.85 for $90 \%$ or $80 \%$ power, respectively.

The key quantity in this computation is $k$, which measures the degree of between community variation. Unfortunately, prior data on $\mathrm{k}$ are seldom available, since this would require prospective studies of incidence in the study population before the design is finalised, and these are likely to be too costly and time consuming. In the Mwanza and Masaka trials, the proposal was based on a plausible estimate of $k(0 \cdot 25)$, implying that HIV incidence rates in each arm varied roughly between $\lambda_{i} \pm 2 k \lambda_{i}$, or from $0 \cdot 5 \lambda_{i}$ to $1 \cdot 5 \lambda_{i}$. Smaller values of $k$ may be attained if the communities are large and chosen to be homogeneous, as in the Rakai study.

If prior data are available on HIV prevalence by community, a further option would be to assume that the coefficient of variation of prevalence is similar to that of incidence, and analysis of data from the east African studies is now in progress to evaluate this assumption. Preliminary data from Rakai suggest a $k$ of 0.16 based on baseline HIV prevalence.

\section{SIZE AND DEFINITION OF COMMUNITIES}

On purely statistical grounds, a large number of small clusters is preferred to a small number of large clusters, since this reduces the design effect resulting from between community variation and increases power for a given total sample size. An absolute minimum of four or five communities are required per arm, to provide sufficient replication to reach useful conclusions. However, there are two reasons for preferring large communities to small communities. Firstly, this may reduce the level of contamination bias resulting from contact of the study population with other untreated communities, which could dilute the observed impact. Secondly, between community variability ( $k$ ) may be reduced when larger clusters 
are used, and data from the east African studies will be analysed to assess this.

In the Mwanza trial, study communities were relatively large (average population around 25000 ) and widely separated, so that travel between communities was time consuming. The study cohort consisted of 1000 people sampled from an "inner zone" within 90 minutes' walk of a central health unit, in order to reduce contamination. In Masaka, the randomisation unit was a "parish", with a population of about 2800. The study cohort was chosen from the three to five villages closest to the health centre, again to reduce contamination. In Rakai, small villages were grouped into "super clusters" (average population around 1200), which were carefully chosen on the basis of social and sexual network studies, so that most sexual contacts were expected to occur within super clusters. Wherever possible, natural barriers such as swamps, hills, and forests were used to provide "buffer zones" between neighbouring super clusters.

\section{MATCHING AND STRATIFICATION}

With relatively few units of randomisation, there is a high probability of some imbalances between the trial arms which may influence the results. To minimise this problem, all three trials employed forms of community matching. For example, in the Mwanza trial, six pairs of communities were formed, matched on type of community (roadside, island, rural) and geographical location, and one community in each pair was randomly allocated to the intervention. In Masaka, six matched triplets of parishes were allocated to the three treatment arms. In Rakai, the 10 study communities were grouped into three strata on the basis of projected HIV prevalence, and randomisation performed within strata.

Matching is only effective if the matching factors are closely correlated with the primary endpoint. In the HIV trials, matching on earlier HIV incidence would be the approach of choice, but this was not feasible. Instead, communities were matched on the basis of factors known from previous studies in the areas to be correlated with HIV prevalence. Baseline data from the Mwanza and Masaka trials ${ }^{21} 22$ confirmed that the matching was highly effective in reducing between community variation in HIV prevalance and thus increasing study power. In Mwanza, the coefficient of variation $\mathrm{k}$ in HIV prevalence was 0.49 over all 12 communities, but only 0.28 when computed within pairs. In Masaka, a $k$ of 0.30 was reduced to 0.19 when analysed within matched triplets (data from first 12 parishes). The power of these studies was increased considerably by the matched designs. Matching was of less importance in the Rakai trial, where selection of more homogeneous communities gave a lower value of $\mathbf{k}$ at baseline $(0 \cdot 16)$.

ANALYSIS OF COMMUNITY RANDOMISED TRIALS A community randomised design requires an appropriate analysis that takes account of between community variation. Methodology for such trials is still under development, but a number of approaches have been reviewed and compared. ${ }^{23-25}$

Two approaches are available that appear robust to small numbers of clusters, and which also have the advantage of simplicity. These involve computing an overall incidence (or prevalence) rate for each community, and analysing these community level outcomes using the $t$ test $^{24}$ (after log transformation if appropriate) or randomisation test. ${ }^{25}$ These methods may be modified to provide confidence intervals as well as significance tests. At least six pairs of communities are required to allow significance at $\mathrm{p}<0.05$ on a two sided randomisation test, or four or five communities per arm with an unmatched or stratified design. The published report of the Mwanza trial used the paired $t$ test. ${ }^{8}$

\section{ADJUSTMENT FOR CONFOUNDERS}

Because of the small number of randomisation units, imbalances between treatment groups are more likely in community randomised trials than in standard clinical trials. It is therefore important to identify potential confounding factors, both at the individual and community level, and to adjust for them in the analysis. The robust methods of analysis discussed above may be modified to adjust for confounders. ${ }^{25}$ In the Mwanza trial, ${ }^{8}$ for example, a logistic regression model was fitted using data on individuals, and including terms for the matched pair, age group, and each of the identified confounding factors. Observed and expected values from the regression model were used to compute an adjusted rate ratio (RR) of HIV seroconversion for each pair, and the $\log (R R)$ were analysed using the paired $t$ test as before.

Criteria for selection of confounders for adjustment have been widely discussed, ${ }^{26} 27$ and a number of alternative strategies have been recommended. In the Mwanza trial, potential confounders were defined as factors known from previous studies in the area to be correlated with HIV prevalence, and which showed a substantial difference between treatment groups in the baseline survey. ${ }^{21}$ Analytical plans were prepared before the end of the trial, listing the factors that would be adjusted for in the final analysis. In HIV intervention trials, a factor that may be of particular importance as a potential confounder is the community HIV prevalence at baseline, because it is likely to be correlated with HIV incidence. It is recommended that future trials adjust for baseline prevalence regardless of the degree of imbalance, preferably by means of regression adjustment. In Mwanza, this resulted in modification of the adjusted efficacy from $42 \%$ to $38 \% .{ }^{28}$

\section{LINKING HIV IMPACT TO STD EFFECTS}

To establish the plausibility of any impact of STD treatment on HIV incidence, it is important to demonstrate that the intervention reduced the incidence and prevalence of target STDs. Analysis of the Mwanza data, with 
adjustment for baseline prevalences, show a substantial impact on active syphilis in both sexes, and on symptomatic urethritis in males. ${ }^{16}$ After one treatment round, preliminary data from Rakai show significant declines in syphilis, trichomonas, and chlamydia, ${ }^{29}$ with additional evidence of declines in severe bacteral vaginosis and gonorrhoea, although there was no decline in reported symptomatology or symptom duration.

Further evidence of a link with STD effects may be obtained by analysing the association between HIV seroconversion and the occurrence of STDs during the period before seroconversion-for example, by means of a nested case control analysis. ${ }^{30}$ This approach suffers from the usual limitations of observational studies, including difficulties in controlling fully for confounding by sexual behaviour. ${ }^{9}$ In addition, it focuses on the cofactor effects of STDs on susceptibility to HIV, but cannot fully capture effects on infectiousness unless partner HIV and STD status is known.

Key messages

- Sample size computations must take account of the community randomised design.

- A minimum of four communities per treatment arm are required.

- Study power may be increased by matching or stratification of communities before randomisation.

- Statistical analysis must take account of the community randomised design. A simple method is to apply the $t$ test to the community level outcomes.

- Adjustment should be made for confounders, including baseline HIV prevalence.

- Reliable data should be collected on the prevalence of STDs to provide supporting evidence for the mechanism of any effect on HIV incidence.

\section{Diagnostic methods}

In trials assessing the impact of STD control measures, it is clearly desirable to obtain comprehensive data on STD prevalence and incidence. In this section we focus on methods for the diagnosis of STDs and other RTIs in population based research trials, rather than diagnostics for use in routine service provision. The ideal STD diagnostic test would require no invasive sample collection or genital examination, would be inexpensive, simple to perform, highly sensitive and specific, would provide immediate results and would discriminate past from present infections. In the absence of such diagnostic ideals, programmes make difficult tradeoffs with respect to acceptability, cost, and the speed and accuracy of diagnosis. This section reviews some of the available methods that may be used in field surveys.

SELF REPORTED SYMPTOMS

Using a structured questionnaire, individuals are asked about current or previous STD/RTI symptoms. Unfortunately, self reported symptoms correlate poorly with laboratory diagnosed infection, and cannot be used as a reliable marker of prevalence. In rural Mwanza, for example, only $15 \%$ of men with laboratory proved gonorrhoea or chlamydia reported urethral discharge at interview. ${ }^{13}$ Moreover, reported symptoms do not always correlate closely with signs observed on clinical examination. ${ }^{14}$ In Rakai, reported symptomatology had very low positive predictive values for any given STD, ${ }^{31}$ and on follow up reported symptoms have not proved useful in tracking laboratory diagnosed STD declines in the intervention arm compared with the control. However, data on self reported symptoms, in combination with laboratory diagnosed infection, may be useful to measure the prevalence of symptomatic STDs.

\section{CLINICAL EXAMINATION}

Clinical observation is inexpensive and technologically simple, but requires well trained workers and adequate privacy and space; the latter often precludes home based examination. If subjects are asked to attend a clinic for examination, findings may be biased unless response rates are high, since asymptomatic individuals may be less willing to undergo genital examination. Furthermore, clinical observation does not appear to provide a sensitive or specific diagnosis of many STDs ${ }^{1032}$; for example, a high proportion of men with laboratory proved gonorrhoea or chlamydia have no clinical signs. ${ }^{13}$ Data from Mwanza indicate substantial variability between clinicians in signs recorded.

LABORATORY TESTS: SPECIMENS COLLECTED BY HEALTH WORKER

In view of the above constraints, laboratory tests are needed to obtain reliable data on STD prevalence.

\section{Vaginal and endocervical specimens}

Specimens collected in field clinics may be used to diagnose vaginal and endocervical infections, although referral to the clinic may reduce compliance and hence the generalisability of results.

Wet mount and saline preparation for trichomonas diagnosis require immediate microscopic examination, and sensitivity is suboptimal. ${ }^{33}$ The Rakai project conducts trichomonas culture using the TV InPouch kit (BioMed Diagnostics, San Jose, CA, USA), ${ }^{34} 35$ which is inoculated in the field and subsequently read in a field laboratory. Although the cost (approximately US\$2) is higher than for wet mount, advantages of the kit include ease of use, high sensitivity and specificity, 3536 and feasibility of home based specimen collection (see below).

Candida albicans can be diagnosed by wet mount, but microscopic examination may miss up to half of culture positive symptomatic candidiasis. ${ }^{37}$ Candida culture remains the gold standard, but is impractical in rural field studies. Colorimetric and other quantitative tests to diagnose candidiasis from vaginal swab 
are currently under development ( $R$ Borchardt, BioMed Diagnostics, personal communication, August 1996).

Bacterial vaginosis has been routinely diagnosed on the basis of vaginal discharge, vaginal $\mathrm{pH}, \mathrm{KOH}$ test for fishy amine odour and clue cells; testing is conducted immediately on fresh samples. More recently, Gram stained slides, scored for the relative proportions of lactobacillus and anaerobic Gram negative organisms (scores 1-10), have been validated for diagnosis of bacterial vaginosis ${ }^{38}$; the technique has the advantages of producing a stable slide for future reading and quality control, and allows grading of severity. Experience in Rakai shows that technicians in peripheral laboratories can readily be trained to read the Gram stained slides (correlation between field and reference laboratory scores $r=0.75$ ).

For chlamydia diagnosis, use of antigen capture enzyme immunoassay (IDEIA, NovoNordisk Diagnostics, Cambridge, UK) proved feasible in the Mwanza study. Antigen detection of chlamydia is less sensitive and specific than DNA amplification by LCR (ligase chain reaction) or PCR (polymerase chain reaction), but is less expensive.

For gonorrhoea diagnosis, Gram stain is of limited use in women, with a sensitivity of $50-70 \%$ in both symptomatic and asymptomatic cases. ${ }^{32}$ Culture on selective media detects over $80 \%$ of infections, ${ }^{32}$ and is necessary to assess drug sensitivity of endemic strains, but collection and transport of specimens are problematic in rural field studies.

\section{Urethral specimens in males}

In the east African trials, urethral specimens have been used for the diagnosis of gonorrhoea (Gram stain, culture) and chlamydia (antigen detection). In males with symptomatic gonococcal urethritis, Gram stain is both sensitive $(90 \%+)$ and specific $(95 \%)$, but in asymptomatic cases sensitivity may be much lower (50-70\%). ${ }^{32}$ Collection of urethral specimens may be problematic since asymptomatic men may not consent to swabbing, or specimens may be taken inadequately because of pain. In the Mwanza trial, swabs were successfully collected at the field clinic from men reporting or observed to have urethral discharge, and from those with a positive leucocyte esterase test (see below). ${ }^{13}$

\section{Serum specimens}

Syphilis represents a key target for STD control efforts and diagnosis via non-treponemal tests (VDRL, RPR, TRUST, New Horizons, Columbia, MD, USA) and treponemal tests (such as TPHA, FTA) is technically feasible. However, the tests are poor at discriminating between new (and presumably more highly transmissible) and old infections. Also, following successful treatment, RPR titres can fall slowly (over many months), complicating programme monitoring and patient management. $^{32}$

New serological assays for Haemophilus ducrey $3^{39-42}$ indicate exposure to the pathogen, but suffer from limitations in sensitivity and specificity, and the duration of a persistent antibody response following resolution of infection has not yet been established. Currently, these assays can only provide epidemiological data on exposure and on the potential importance of chancroid in the population.

Herpes simplex virus (HSV) serology is of limited use in the diagnosis of acute infection, but type specific tests can indicate recent seroconversion and previous exposure. In community based trials, HSV-2 serostatus may be used as an epidemiological marker of sexual risk behaviour, particularly in younger age groups. ${ }^{434}$

Serological specimens can be collected by trained workers in both household and clinic settings. While compliance with venous blood collection in the east African trials has generally been high, there is often resistance to providing blood specimens in community surveys, particularly when requested repeatedly over several years.

\section{Specimens from genital ulcers}

Scrapings and swabs from genital ulcers can be used for the diagnosis of HSV-2 (viral antigen detection) and syphilis (dark field microscopy). Specimens for HSV antigen detection can be transported to the appropriate laboratory, but dark field examination needs to be conducted immediately and requires a practised technician. $H$ ducreyi can be cultured from chancroid ulcer swabs; however, such culture is technically difficult, has been implemented successfully in only a few laboratories in Africa, and is not feasible in most field trials. A multiplex $\mathrm{PCR},{ }^{45}$ available for research purposes (Roche Molecular Systems, Branchburg, NJ, USA), is being used in Rakai to differentiate between lesions caused by syphilis, chancroid, and HSV-2.

Collection of genital ulcer swabs is hampered by the fact that many ulcers remain unnoticed (particularly in women) and because subjects may be reluctant to accept scraping. However, ulcer swabs are useful in determining the predominant pathogens causing symptomatic ulcers in a given population.

LABORATORY TESTS: SPECIMENS COLLECTED BY STUDY PARTICIPANT

Urine specimens

Given the limited feasibility of genital examination in household surveys, there are advantages to non-invasive specimen collection by study participants. In the Rakai project, over $94 \%$ of male and female participants agreed to provide a urine specimen, collected in the home at the time of interview.

The urinary dipstick leucocyte esterase test (LET), is used to diagnose male urethritis based on esterase produced by polymorphonuclear leucocytes. In the United States, this test has high specificity but low sensitivity. ${ }^{46}$ In Mwanza, there is a high rate of false positive LET results, possibly due to the high prevalence of Schistosoma haematobium in the region. ${ }^{1047}$ The utility of LET as a survey tool is thus contingent on the rates of gonorrhoea, 
chlamydia, and other urinary tract pathogens in the population.

In the Rakai project, urine specimens from both males and females are tested by the ligase chain reaction (LCR, Abbott Laboratories, Abbott Park, IL, USA) for gonorrhoea and chlamydia. These tests have high sensitivity and specificity when evaluated against culture. ${ }^{48-51}$ LCR is currently expensive and requires specialised technology, but specimens can be collected in the home, processed in a small field laboratory, frozen, stored for long periods of time, and shipped to a reference laboratory for analysis. In low prevalence populations, it may be possible to reduce costs through pooling of specimens.

\section{Self administered vaginal swabs}

In Rakai, $95 \%$ of women have agreed to and correctly provided two self administered vaginal swabs collected in the home at the time of interview. In the home, one swab is immediately inoculated into the TV InPouch culture kit, and used to assess trichomonas prevalence. ${ }^{34}{ }^{35}$ The second swab is used to prepare an air dried slide, which is subsequently Gram stained and scored for bacterial vaginosis. ${ }^{38}$

In conclusion, self administered specimen collection has major advantages in terms of population acceptability. Gonorrhoea, chlamydia, trichomonas, and $\mathrm{BV}$ can be diagnosed through urine and vaginal swabs collected by the participant in the home. By combining serological specimens with urine and self administered vaginal swabs, the Rakai project has been able to collect reliable data on population rates of most key STDs. Disadvantages of this methodology include cost (LCR is expensive and many uninfected people are screened), and delays in receiving some results (LCR samples are sent to a reference laboratory in the United States for testing). These disadvantages are offset by an ability to assess more reliably the effects of community based interventions and, in the future, to monitor ongoing programmes through sample surveys.

Key messages

- Self reported symptoms and clinical signs correlate poorly with laboratory diagnosed STD infections.

- Tests can be performed on vaginal or urethral swabs, but specimen collection and some test procedures can be difficult under field survey conditions.

- Self administered specimen collection has major advantages in terms of population acceptability.

- Urine specimens can be frozen and tested for gonorrhoea and chlamydia using LCR or PCR.

- Self administered vaginal swabs can be tested for trichomonas using the TV InPouch culture kit, and can be Gram stained and scored for bacterial vaginosis.

- Sera can be tested for syphilis and $H$ ducreyi, although available tests are subject to some limitations. HSV2 serology can be used as a marker of sexual risk behaviour especially in younger age groups.

\section{Treatment regimens}

Given the resource constraints under which health services operate in most developing countries, only the treatment of bacterial and protozoal infections was discussed at the workshop. Antiviral therapy was not considered.

An ideal treatment regimen for an STD would be single dose, oral, highly effective and likely to remain so, free from significant side effects, inexpensive, and effective against more than one pathogen. In presumptive (that is, syndromic or mass) treatment, medications are administered to people who may not be infected, so it is particularly important that treatment regimens should be safe. Ideally all treatments should be inexpensive, but trials of initially more expensive drugs may be warranted to demonstrate efficacy, with the understanding that many drug prices are rapidly declining. Means of monitoring for and avoiding selection of drug resistant STD or enteric organisms also need to be considered with all regimens, particularly those which require multidose therapy.

Standard treatment regimens recommended by WHO are reviewed elsewhere, ${ }^{52}$ and this report focuses on the modified treatment strategies implemented in the three east African trials: the syndromic treatment algorithms used in Mwanza and Masaka, and the mass treatment regimen used in Rakai.

\section{SYNDROMIC TREATMENT IN THE MWANZA AND} MASAKA TRIALS

Syndromic treatment is based on a presumptive diagnosis of genital ulcer, genital discharge, or other STD syndromes. WHO recommends treatment of STD syndromes using highly effective regimens at the first visit, because this is cost effective in terms of sequelae prevented and future infections averted. This approach is expensive owing to high drug costs, and an alternative strategy is to use a cheaper regimen as first line treatment, reserving more expensive and effective regimens for treatment failures. Such an approach, which may be appropriate in settings with ready access to services for repeat visits, was adopted in the Mwanza and Masaka studies. The selection of appropriate first line drugs should depend on the pattern of resistance in a given population.

The following syndromic approaches were used in the Mwanza trial.

\section{Genital ulcer syndrome (GUS)}

The most frequent bacterial causes of GUS are chancroid and syphilis. To cover these diseases, the first line regimen was trimethoprimsulphamethoxazole $320 \mathrm{mg} / 1600 \mathrm{mg}$ twice daily for 2 days for chancroid and benzathine penicillin 2.4 million units intramuscularly for syphilis. If the ulcer persisted for 7 days, second line treatment was trimethoprimsulphamethoxazole $320 \mathrm{mg} / 1600 \mathrm{mg}$ twice daily on alternate days $\times 3$, and persistent treatment failures after a further 7 days were given ciprofloxacin $250 \mathrm{mg}$ twice daily for 3 days. 
Vaginal discharge

It is wasteful of resources to treat all women complaining of vaginal discharge for all common causes of this condition (trichomonas, candidiasis, bacterial vaginosis, gonorrhoea, and chlamydia). If examination with a vaginal speculum is possible, candidiasis can be presumptively diagnosed clinically. If, in addition, a microscope is available, $T$ vaginalis infection and bacterial vaginosis can also be diagnosed. Unfortunately, no simple tests are available to diagnose gonorrhoea and chlamydial infection in women. Moreover, several studies have shown that the presence of these infections is poorly correlated with the complaint of vaginal discharge.$^{10}{ }^{17}$ In order to reduce the level of overtreatment, several attempts have been made to identify those women at high risk of gonorrhoea or chlamydial infection-either by asking simple questions on risk factors ${ }^{10} 17$ or by speculum examination for evidence of mucopurulent cervicitis. $^{53}$ Unfortunately, none of these simple methods has a sensitivity and specificity above $70 \%$, and predictive value is poor.

The following algorithms based on the appearance of the discharge were used in Mwanza:

(i) Appears like thrush (that is, Candida): gentian violet.

(ii) Does not appear like thrush: assume discharge due to gonorrhoea, chlamydia, trichomonas, or bacterial vaginosis and treat with first line trimethoprim-sulphamethoxazole $400 \mathrm{mg} / 2000 \mathrm{mg}$ twice daily for 2 days plus doxycycline $100 \mathrm{mg}$ twice daily for 7 days plus metronidazole $2 \mathrm{~g}$ immediately. If discharge persists for 7 days, the second line treatment was doxycycline $100 \mathrm{mg}$ twice daily for 7 days, and if still unresolved, ciprofloxacin $500 \mathrm{mg}$ immediately.

\section{Urethral discharge}

Men complaining of urethral discharge were treated presumptively for gonorrhoea and chlamydia. First line treatment was trimethoprim-sulphamethoxazole $\quad 400 \mathrm{mg} / 2000 \mathrm{mg}$ twice daily for 2 days plus doxycycline $100 \mathrm{mg}$ twice daily for 7 days. Second line treatment was metronidazole $2 \mathrm{~g}$ immediately plus doxycycline $100 \mathrm{mg}$ twice daily for 7 days, and third line treatment ciprofloxacin $500 \mathrm{mg}$ immediately.

\section{Pelvic inflammatory disease (PID)}

Suspected PID (based on a complaint of lower abdominal pain) was treated presumptively for gonorrhoea, chlamydia, and anaerobic infections. The first line treatment was trimethoprim-sulphamethoxazole $400 \mathrm{mg} / 2000$ $\mathrm{mg}$ twice daily for 2 days plus doxycycline $100 \mathrm{mg}$ twice daily for 14 days plus metronidazole $400 \mathrm{mg}$ twice daily for 7 days. Second line treatment was ciprofloxacin $500 \mathrm{mg}$ immediately.

Treatment algorithms in Masaka are broadly similar, the main differences being use of chloramphenicol in place of trimethoprim-sulphamethoxazole for genital discharge and PID, and erythromycin for second line treatment of genital ulcers.

MASS TREATMENT IN THE RAKAI TRIAL

The mass treatment regimen of the Rakai trial combines single oral doses of azithromycin $(1 \mathrm{~g})$ and ciprofloxacin $(250 \mathrm{mg})$. Each drug alone is highly effective against gonorrhoea and chancroid ${ }^{54-57}$; the combined regimen reduces the risk of drug resistance and directly observed therapy ensures compliance. Azithromycin is also highly effective against chlamydia. 5558 Ongoing monitoring has revealed no gonorrhoea resistance to azithromycin or ciprofloxacin in samples from people with genital discharge attending Rakai clinical facilities. Under $10 \%$ of subjects in the mass treatment arm report side effects, mainly mild gastrointestinal upset which resolves within 2 days. The cost of this drug combination has fallen substantially over the past 2 years. In pregnant women, ciprofloxacin is replaced by cefixime, $400 \mathrm{mg}$ immediately, which is also highly effective against gonorrhoea. ${ }^{59}$

A single dose of metronidazole $(2 \mathrm{~g})$ is given 1 day later for mass treatment of trichomonas and bacterial vaginosis. This regimen shows high cure rates (over $85 \%$ ) for trichomonas, an efficacy of over $70 \%$ one month after treatment for bacterial vaginosis, ${ }^{32}$ and has been shown to be safe during pregnancy. ${ }^{60}$ In Rakai, this dose was associated with a reduced proportion of women with severe bacterial vaginosis in the intervention arm compared with the control arm 9 months after treatment. ${ }^{29}$ However, bacterial vaginosis tends to recur, as has been found by other researchers following both single and multidose treatments. ${ }^{61}$

There is no established single dose oral treatment for syphilis, so intramuscular benzathine penicillin ( 2.4 million units) is given to all intervention arm subjects with serological evidence of syphilis (based on a TRUST test run in the field laboratory). Penicillin is provided in the home within 24 hours after serological testing. Some observations suggest that azithromycin may also be effective against syphilis, ${ }^{62}$ but the available data refer to a 10 day course and shorter regimens remain to be evaluated.

\section{DRUG RESISTANCE}

All STD treatment strategies entail the potential of selecting for bacterial strains with antibiotic resistance. This is of particular concern with multidose treatment regimens and with mass treatment, and for this reason the Rakai trial has used single dose, directly observed treatment and multidrug combinations. The development of resistance may be more difficult to control in the context of routine services, where periodic shortages of drug supplies, or use of drugs of subobtimal quality, may give rise to partial treatment.

Single dose ciprofloxacin is often a component of STD treatment regimens, and while this is unlikely to promote resistance in target organisms such as Neisseria gonorrhoeae, ${ }^{63}$ there is a possibility of promoting resistance in non- 
target organisms such as Escherichia coli, Salmonella, and Shigella species. ${ }^{64}$ As this is a potential adverse effect of mass treatment or syndromic treatment interventions, trials should monitor for the development of antibiotic resistance. It is reassuring that weekly doses of single oral dose azithromycin are now used routinely for prophylaxis against disseminated Mycobacterium avium (MAC) infection in HIV infected individuals, without the emergence of drug resistance. ${ }^{65}$

\section{Key messages}

- Syndromic treatment involves treatment of clinical syndromes for the pathogens mainly responsible for those syndromes.

- Depending on resources, WHO treatment algorithms can be adapted to use a cheaper regimen for first line treatment, reserving more expensive and effective regimens for treatment failures.

- Selection of drugs requires data on patterns of resistance in a given population.

- Treating vaginal discharge is problematic because limitations in diagnostic and risk score methods complicate diagnosis of cervical infections. Simple, cheap, and reliable diagnostics for gonorrhoea and chlamydia in women are an urgent priority.

- Mass treatment should employ single dose oral regimens of proved safety and high efficacy against important STD pathogens.

- Trials should monitor for the development of antibiotic resistance in target and nontarget organisms.

\section{Ethical issues}

Community based trials of STD treatment involve a number of complex ethical issues.

\section{CONTROL GROUPS}

Given the current state of knowledge concerning the efficacy of condoms in preventing HIV and STDs, the increased risk of HIV transmission associated with STDs, and the results of the Mwanza intervention trial, what services should be provided in the intervention and control groups in these trials?

Workshop participants agreed that the promotion of condoms and voluntary HIV testing and counselling should be carried out in a culturally sensitive manner in all trial communities. STD treatment provision in control groups is a more difficult issue, which requires careful consideration and discussion with national authorities. Enhanced treatment services are likely to have different effects in different populations. If the purpose of a randomised trial is to determine the impact of providing enhanced services over and above those currently offered in a specific population, then it may be appropriate for the control group to receive the current "standard of care" in that population. An important principle is that control communities should not be disadvantaged by taking part in the trial. However, substantially improved provision in the control group could invalidate the findings of the study. It was recommended that STDs detected in the control arm during field surveys should, at the very least, be referred to appropriate services for treatment.

CONSENT, HIV TESTING, AND NOTIFICATION Informed consent, either verbal or written, should be obtained from all participants. Subjects should be aware that not all communities will be receiving the experimental intervention, and that they will be tested for HIV and other STDs. They should also be aware that they can withdraw from the study at any time and that their HIV status will be kept strictly confidential. It is important to avoid creating any false expectations about the intervention; in particular, subjects should be discouraged from increasing their risk behaviour because they believe that they may be receiving an intervention against HIV. Incentives, whether cash or in kind, should generally be avoided, although it may be appropriate to compensate subjects for time and other costs relating to survey participation.

Services for voluntary HIV testing, together with pre- and post-test counselling, should be made available to all trial participants. However, restricting trial participation to those individuals who want their results could lead to self selection bias and poor coverage. It would clearly be wrong to insist that all participants be given their HIV results; notification should not be compulsory or coerced, given the risk of domestic violence. ${ }^{66}$ Although subjects should be encouraged to bring their partners for simultaneous testing, this must also be voluntary. If the investigators are aware of discordant couples, protocols for HIV test notification should follow national guidelines. Workshop participants noted that the balance between responsibility to the individual patient and to the community is beginning to shift perceptibly in the industrialised countries, with the recognition that individuals need to make decisions, such as those related to counselling, on their own. Contact tracing and partner notification for STDs should ideally be carried out by the patient rather than the programme, in order to avoid stigmatisation and low compliance because of fear. Such an approach is both ethically more sound, and more practical in the rural African setting.

\section{STD SCREENING}

The ethical issues associated with STD screening depend upon the setting. If mass treatment is given after specimens are taken then the results are of no real clinical benefit. If syndromic treatment is provided, then there will be no specimens. However, if screening specimens are taken and the results are available quickly, as in RPR screening for active syphilis, then treatment must be made available. A difficult situation arises if specimens are taken but not tested for some months or years. What moral imperative arises to treat infections identified long after the event? The appropriate action probably depends on the infection: for example, the average duration of 
gonorrhoeal infection may be as little as 6 weeks even if untreated, while syphilis may persist for 30 years or more. If screening for STDs is performed, and is to be followed by targeted treatment of infected individuals, then this should be made clear in the informed consent procedure.

\section{INTERIM ANALYSES}

Because of their cost and complexity, community randomised trials are unlikely to have the power to detect important differences early in the course of the study. Frequent interim analyses should therefore be discouraged, since they may result inappropriately in the premature termination of the trial. Such partial trial results are often too imprecise to be of conclusive value, implying that trial subjects may have been subjected to inconvenience and risk for no societal benefit.

Where interim analyses are to be conducted, they should be carefully planned from the outset and incorporated in the study protocol. Issues that need to be considered include: what are the criteria to initiate an interim analysis, what are the guidelines for action, and what actions are available? For example, many more or many fewer cases than expected may trigger an interim analysis to ensure that an unexpectedly effective intervention, or unexpected adverse effects, are identified as early as possible. The implications of repeated analysis for significance testing at the end of the trial also require consideration.

Safety and effectiveness can generally be monitored without breaking treatment codes. Monitoring committees should be independent of the investigators, and should ensure that, as far as possible, blindness of the investigators is maintained.

\section{APPROPRIATE INTERVENTIONS}

A key question is whether a proposed experimental intervention should necessarily be affordable and sustainable in the setting in which it is to be evaluated. Ideally, any intervention should be affordable and sustainable, but circumstances change, drug costs are not immutable, and policy makers need information about evaluated and costed interventions in order to make rational decisions.

This is a particular issue for mass treatment interventions, which are likely to be costly. However, if asymptomatic STDs play an appreciable role in rapid reinfection with STDs and in facilitating HIV transmission, this approach may be cost effective in comparison with syndromic treatment, both as an STD and an HIV intervention. If this can be confirmed, and given the seriousness of the present global HIV pandemic, such an option may conceivably be attractive to policy makers. There are precedents: annual mass treatment with ivermectin for onchocerciasis is currently planned for 16 million people in 16 African countries at a cost of US\$25-30 million per year.
Key messages

- The provision of STD treatment and other HIV prevention measures in control groups require careful consideration.

- Informed consent should be obtained from all participants, and confidentiality of test results must be ensured.

- Services for voluntary HIV counselling and testing should be available to all participants. Disclosure of HIV test results should be voluntary.

- Wherever STD test results are available rapidly, treatment should be provided both in the intervention and control groups.

- Interim analyses should be carefully planned and blindness of the investigators maintained. Inappropriate premature termination of trials should be avoided.

- Experimental interventions should be potentially affordable and sustainable in the study population, taking account of likely reductions in drug costs over time.

We wish to thank the Rockefeller Foundation, the National Institutes of Health, the Fogarty International Postdoctora AIDS Research Training Program, and the Hewlett Fund fo financial support for the workshop. We are grateful to the John Hopkins School of Hygiene and Public Health for hosting the workshop, and to secretarial staff of the Departments of Population Dynamics and International Health for providin support services. Special thanks are due to Maria Zanella for excellent administrative support.

\section{HIV/STD trials workshop group}

All workshop participants contributed to the technical discussions and to the material presented in this paper. The following is a full list of participants: John Bartlett, Ron Brookmeyer, Gilbert Burnham, Charlotte Gaydos, Ron Gray, Neal Halsey, Richard Morrow, Larry Moulton, Tom Quinn, Anne Rompalo, Jonathan Zenilman (Johns Hopkins University (JHU), Baltimore); Lucy Carpenter, Jane Kengeya-Kayondo, Jimmy Whitworth (MRC Programme on AIDS in Uganda, Entebbe); Heiner Grosskurth, Richard Hayes, David Mabey (London School of Hygiene \& Tropical Medicine (LSHTM)) Sharon Hillier (University of Pittsburgh); Penny Hitchcock, Zeda Rosenberg (National Institutes of Health, Bethesda) Noah Kiwanuka (Uganda Virus Research Institute, Entebbe) Philippe Mayaud, Jim Todd (LSHTM and African Medica and Research Foundation); Maria Wawer (Columbia University, New York and JHU)

1 The Status and Trends of the Global HIV/AIDS Pandemic Symposium Final Report, Vancouver, 5-6 July 1996 Harvard, MA: AIDSCAP/Family Health International,

2 Mann J, Tarantola DJM, Netter TW, eds. AIDS in the world. Cambridge, MA: Harvard University Press, 1992.

3 Laga M, Diallo MO, Buvé A. Inter-relationship of sexuall transmitted diseases and HIV: where are we now? AIDS 1994;8 (suppl 1):S1 19-24.

4 Wasserheit $\mathrm{N}$. Interrelationships between human immun odeficiency virus infections and other sexually transmitted disease. Sex Transm Dis 1992;19:61-77.

5 DeSchryver A, Meheus A. Epidemiology of sexually transmitted diseases: the global picture. Bull WHO 1990;68 mitted dis

6 Over M, Piot P. HIV infection and sexually transmitted diseases. In: Jamison DT, Mosley WH, Measham AR Bobadilla $\mathrm{JL}$, eds. Disease control priorities in developing countries. New York: Oxford University Press, 1993: 455-527

7 World Bank. World development report 1993: investing in health. New York: Oxford University Press, 1993.

8 Grosskurth $\mathrm{H}$, Mosha F, Todd J, et al. Impact of improved treatment of sexually transmitted diseases on HIV infection in rural Tanzania: randomised controlled trial. Lancet 1995;346:530-6.

9 Mertens T, Hayes RJ, Smith PG. Epidemiologic methods to study the interaction between HIV infection and other sexually transmitted diseases. AIDS 1990;4:57-65.

10 Mayaud P, Grosskurth H, Changalucha J, et al. Risk assessment and other screening options for gonorrhoea and chlamydial infections in women attending rural chlamydial infections in women attending rural

11 Mulder D. Disease perception and health-seeking behaviour for sexually transmitted diseases. In: Prevention and management of sexually transmitted diseases in eastem and southern Africa: current approaches and future directions. Nairobi: NARESA Monograph No 3, NARESA, PO 11771, Nairobi, 1994

12 Newell J, Senkoro K, Mosha F, et al. A population-based 
study of syphilis and sexually transmitted disease syndromes in north-western Tanzania. 2. Risk factors and health seeking behaviour. Genitourin Med 1993;69:421-6.

13 Grosskurth H, Mayaud P, Mosha F, et al. Asymptomatic gonorrhea and chlamydia infection in rural Tanzanian men. $B M F$ 1996;312:277-90

14 Buvé $\mathrm{A}$, Mosha $\mathrm{F}$, Watson-Jones $\mathrm{D}$, et al. Is asymptomatic urethritis in men an obstacle to effective STD control? A community study in Mwanza, Tanzania. XI International Conference on AIDS, Vancouver, July 1996, abstract No C 341 .

$15 \mathrm{Ka}$-Gina G, Grosskurth H, Mwijarubi E, et al. The feasibility of integrating STD management into a rural primary health care system: results from a large scale intervention study in Mwanza, Tanzania. 11th Meeting of Interstudy in Mwanza, Tanzania. 11th Meeting of Inter-
national Society for STD Research, New Orleans, national Society for STD

16 Mwijarubi E, Grosskurth H, Mosha F, et al. Improved STD treatment significantly reduces prevalence of syphilis and symptomatic urethritis in rural Tanzania. XI International Conference on AIDS, Vancouver, July 1996, abstract No LB C 6062.

17 Vuylsteke B, Laga M, Alary $M$, et al. Clinical algorithms for the screening of women for gonococcal and chlamydial infection: evaluation of pregnant women and prostitutes in Zaire. Clin Infect Dis 1993;17:82-8.

18 Hethcote $\mathrm{HH}$, Yorke JA. Gonorrhea transmission dynamics and control. Lecture notes in biomathematics. New York: Springer, 1984

19 Hayes R, Mosha F, Nicoll A, et al. A community trial of the impact of improved STD treatment on the HIV epidem in rural Tanzania: 1 Design. AIDS 1995;9:919-26.

20 Smith PG, Morrow RH. Field trials of health intervention in developing countries: a tool box. London: Macmillan, 1996.

21 Grosskurth $\mathrm{H}$, Mosha $\mathrm{F}$, Todd J, et al. A community trial of the impact of improved STD treatment on the HIV epidemic in rural Tanzania: 2 Baseline survey results. epidemic in rural Ta

22 Kengeya-Kayondo JF, Nabaitu J, Malamba S, Whitworth J. A trial of IEC alone and with improved STD care on HIV incidence in Uganda: are study communities comparable? XI International Conference on AIDS,

Donner A, Klar N. Methods for comparing event rates in intervention studies when the unit of allocation is a cluster. Am $\mathcal{F}$ Epidemiol 1994;140:279-89.

24 Donner A, Donald A. Analysis of data arising from a stratified design with the cluster as unit of randomization. Stat Med 1987;6:43-52.

25 Gail MH, Mark SD, Carroll RJ, Green SB, Pee D. On design considerations and randomization-based inference for comm

26 Altman DG, Doré CJ. Randomisation and baseline comparison in clinical trials. Lancet 1990;335:49-153.

27 Senn S. Testing for baseline balance in clinical trials. Stat Med 1994;13:1715-26.

28 Hayes R, Grosskurth H, ka-Gina G. Impact of improved treatment of sexually transmitted disease on HIV infection. Lancet 1995;346:1159-60.

29 Wawer M, Sewankambo NK, Gray RH, et al. Communitybased trial of mass STD treatment for HIV control, Rakai, Uganda: preliminary data on STD declines. XI International Conference on AIDS, Vancouver, July 1996, abstract No C443.

30 Laga M, Manoka A, Kivuvu M, et al. Non-ulcerative sexually transmitted diseases as risk factors for HIV-transmission in women: results from a cohort study. AIDS 1993; 7:95-102.

31 Paxton LA, Sewankambo N, Wawer MJ, et al. Asymptomatic genital tract infections in a rural district of Uganda. XI International Conference on AIDS, Vancouver, July 1996, abstract No C340.

32 Holmes KK, Mardh P, Sparling PH, et al, eds. Sexually transmitted diseases. 2nd ed. New York: McGraw-Hill, transmitce 1990.

33 Hillier S, Arko R. Vaginal infections. In: Morse SA, Moreland AA, Holmes KK, eds. Atlas of sexually transmitted diseases and AIDS. 2nd ed. London: Mosby-Wolfe, 1996.

34 Wawer MJ, McNairn D, Wabwire-Mangen F, et al. Selfadministered vaginal swabs for population-based assessment of Trichomonas vaginalis prevalence. Lancet 1995; 345:131-2.

35 Borchardt DA, Smith RF. An evaluation of an InPouch TV culture method for diagnosing Trichomonas vaginalis infection. Genitourin Med 1991;67:149-52.

36 Draper D, Parker R, Patterson E, et al. Detection of Trichomonas vaginalis in pregnant women with the InPouch TV Culture System. F Clin Microbiol 1993;31: 1016-8.

37 Bertholf ME, Stafford MJ. Colonization of Candida albicans in vagina, rectum and mouth. $\mathcal{F}$ Family Pract 1983;16: 919.

38 Eschenbach DA, Hillier SL, Critchlow C, et al. Diagnosis and clinical manifestations of bacterial vaginosis. $A m \mathcal{F}$ Obstet Gynecol 1988;158:819-28.

39 Alfa $M$, Olson $N$, Degagne $P$, et al. Humoral immune response of humans to lipooligosaccharide and outer membrane proteins of Haemophilus ducreyi. $\mathcal{F}$ Infect Dis 1993;167:1206-10.

40 Desjardins $M$, Thompson $R$, Filion LG, et al. Standardisation of an enzyme immuno assay for human antibody to Haemophilus ducreyi. F Clin Microbiol 1992 30:2019-24.

41 Museyi K, VanDyck E, Vervoort $T$, et al. Use of an enzyme immunoassay to detect serum IgG antibodies to Haemophilus ducreyi. F Infect Dis 1988;157:1039-43.

42 Roggen E, Hoofd G, Van Dyck E, Piot P. Enzyme immunoassays (EIAs) for the detection of Haemophilus ducreyi serum IgA, I

43 Cowan FM, Johnson AM, Ashley RL, Corey L, Mindel A. Antibody to HSV2 as a serological marker of sexual Antibody to HSV2 as a serological marker of

44 Wagner HU, Van Dyck E, Roggen E, et al. Seroprevalence and incidence of sexually transmitted diseases in a rura Ugandan population. Int $\mathcal{F}$ STD AIDS 1994;5:332-7.

45 Orle KA, Gates CA, Martin DH, Body BA, Weiss JB. Treponema pallidum and Herpes simplex virus types 1 and 2 from genital ulcer. $\mathcal{F}$ Clin Microbiol 1996;34:49-54.

46 McNagny SE, Parker RM, Zenilman JM, Lewis JS Urinary leucocyte esterase test: a screening method for the detection of asymptomatic chlamydial and gonococthe detection of asymptomatic chlamydial and go

47 Mayaud $\mathrm{P}, \mathrm{Changaluch} \mathrm{J}$, Grosskurth $\mathrm{H}$, et al. The value of urine specimens in screening for male urethritis and its microbial aetiologies in Tanzania. Genitourin Med 1992, 68:361-5.

48 Ching S, Lee H, Hook EW, Jacobs MR, Zenilman J. Ligase chain reaction for detection of Neisseria gonorrhoeae in urogenital swabs. F Clin Microbiol 1995;33:3111-4.

49 Lee HH, Chernesky MA, Schachter J, et al. Diagnosis of Chlamydia trachomatis genitourinary infection in women by ligase chain reaction assay of urine. Lancet 1995; 345:213-6.

50 Buimer M, Van Doornum GJ, Ching S, et al. Detection of Chlamydia trachomatis and Neisseria gonorrhoeae by ligase chain reaction-based assays with clinical specimens from various sites: implications for diagnostic testing and screening. $\mathcal{f}$ Clin Microbiol 1996;34:2395-400

51 Chernesky MA, Lee H, Schachter J, et al. Diagnosis of Chlamydia trachomatis urethral infection in symptomatic and asymptomatic men by testing first-yoid urine in a ligase chain reaction ay. $f$ Infect Dis 1994;170:1308-11.

52 World Health Organisation. Management of sexually trans mitted diseases. (WHO/GPA/TEM/94-1.) Geneva: World Health Organisation, 1994

53 Braddick MR, Ndinya-Achola JO, Mirza NB, et al. Towards developing a diagnostic algorithm for Chlamydia trachomatis and Neisseria gonorrhoeae cervicitis in pregnancy. Genitourin Med 1990;66:62-5

54 Ballard R, Morse S. Chancroid. In: Morse SA, Moreland AA, Holmes KK, eds. Atlas of sexually transmitted diseases and AIDS. 2nd ed. London: Mosby-Wolfe, 1996.

55 Steingrimsson $\mathrm{O}$, Olafsson $\mathrm{JH}$, Throrarinsson $\mathrm{J}$, et al. Azithromycin in the treatment of sexually transmitted disease. $\mathcal{F}$ Antimicrob Chem 1990;25 (suppl A):109-14.

56 Ballard R, Radebe F, Mampuru M, Dangor Y, Fehler HG A comparison of ofloxacin and ciprofloxacin in the treatment of chancroid. Sex Transm Dis 1994;21 (Suppl 2, Abst 110):S189.

57 Echols RM, Heyd A, O'Keeffe BJ, Schacht P. Single-dose ciprofloxacin for the treatment of uncomplicated gonorrhea: a worldwide summary. Sex Transm Dis 1994;21: 345-52.

58 Martin DH, Mroczkowski TF, Dalu AA, et al. A controlled trial of single dose azithromycin for the treatment of chlamydial urethritis and cervicitis. $N$ Engl f Med 1992; 327:921-5.

59 Handsfield HH, McCormack WM, Hook EW, et al. A comparison of single dose cefixime with ceftriaxone as treatment of uncomplicated gonorrhea. $N$ Engl $\mathcal{f}$ Med 1991;325:1337-41.

60 Burtin P, Taddio A, Ariburnu O, Einarson TR, Koren G Safety of metronidazole in pregnancy: a meta-analysis. Am $\mathcal{F}$ Obstet Gynecol 1995;172:525-9.

61 Sweet RL. New approaches for the treatment of bacterial vaginosis. Am f Obstet Gynecol 1993;169:479-82.

62 Verdon MS, Hunter Handsfield H, Johnson RB. Pilot study of azithromycin for treatment of primary and secondary syphilis. Clin Infect Dis 1994;19:486-8.

63 Zenilman JM. Gonococcal susceptibility to antimicrobials in Baltimore, 1988-1994: what was the impact of ciprofloxacin as first line therapy for gonorrhea? Sex Transm Dis 1996;23:213-8.

64 Pena C, Albareda JM, Pallares R, et al. Relationship between quinolone use and emergence of ciprofloxacin resistant $E$ coli in bloodstream infections. Antimicrob Agents Chemother 1995;39:520-4.

65 Havlir DV, Dube MP, Sattler FR, et al. Prophylaxis against disseminated Mycobacterium avium complex with weekly azithromycin, daily rifabutin, or both. $N$ Engl $f$ Med 1996;335:392-8

66 Temmerman M, Ndinya-Achola J, Ambani J, Piot P. The right not to know HIV-test results. Lancet 1995;345 969-70. 